\title{
EL PAPEL DE LAS ADMINISTRACIONES PÚBLICAS EN LA CONFIGURACIÓN DE LAS AUTOPISTAS DE LA INFORMACIÓN DE ANDALUCÍA COMO SOPORTE PARA LOS SISTEMAS DE INFORMACIÓN
}

Jesús Gabriel MORENO NAVARRO*

\section{INTRODUCCIÓN}

Las administraciones central, autonómicas y municipales están participando en la revolución de las nuevas tecnologías de la información (NTI) con desigual interés y fortuna. La propia revolución se está sirviendo de vehículo a sí misma, provocando una situación de atracción y desconcierto donde los intereses mercantiles y políticos pueden primar sobre la lógica de la correcta configuración sobre el territorio de una red de transmisión de datos, la cual debe dar soporte físico a los costosos sistemas de información territoriales que se están desarrollando y poniendo en servicio en Andalucía. El principio constitucional sobre la autonomía de las tres administraciones no exime a éstas de la obligación de ofrecer al ciudadano un conjunto de centros de gestión con una actividad fluida y con sistemas de información intercomunicados que sean capaces de proporcionar al contribuyente unos servicios con la calidad que actualmente se requiere, estando este deber en consonancia con la ley 30/92 del Régimen Jurídico de las Administraciones Públicas y del Procedimiento administrativo común. El propio Estatuto de Andalucía en el artículo $4^{\circ}$ párrafo cuatro obliga a la Comunidad Autónoma a articular la gestión de sus servicios periféricos propios a través de las Diputaciones Provinciales. Por otra parte, dado el carácter articulador que se le reconoce a las telecomunicaciones, Ia operatividad de los sistemas de información en lo que a su topología y componente lógico se refiere está contenida en el capítulo de comunicaciones dentro del cual se incluye el de las telecomunicaciones. Este aspecto se tiene en cuenta dentro de las directrices del Plan Andaluz de Desarrollo Económico y Territorial 1991-94, tal y como se advierte en las Bases para la Ordenación del Territorio de Andalucía BELLOSO y ESPAÑA (1995). A la luz de este contexto

\footnotetext{
* Profesor de Análisis Geográfico Regional. Universidad de Sevilla.
} 
jurídico administrativo, este trabajo pretende desvelar los obstáculos que sobre e terreno se están encontrando los usuarios y gestores de los sistemas de informa ción para cumplir los objetivos marcados. Se expondrá una problemática no nuev: sobre la disfuncionalidad de las administraciones por conflicto o mala interpreta ción de las competencias en materia de infraestructuras y servicios, pero esta ve: con el aliciente del nuevo hito que suponen las Autopistas de la Información, cuyc máximo exponente es la INTERNET y cuya consigna es "no reconocer frontera: para el tráfico de información" facilitando la libre circulación de información : escala mundial. Para concluir se pondrá de relieve la falta de esta visión globa que refleja la INTERNET y que no coincide con la trayectoria que al respecto siguer las administraciones públicas en España y el Informe Bangeman en la concepciór de las políticas de infraestructura telemática e informática, manteniendo una aproximación al tema desde la perspectiva del análisis geográfico.

\section{LA GEOGRAFÍA Y LAS TELECOMUNICACIONES}

Las telecomunicaciones tienen dos aspectos fundamentales abordables desde nuestra disciplina: como objeto de estudio por su plasmación en el territorio y comc herramienta de trabajo. A su vez, el primer aspecto puede estudiarse según su capacidad estructurante y según la lógica de su topología.

\subsection{Impacto de las NTI sobre el territorio}

En el primer aspecto los estudios sobre el impacto de las telecomunicaciones sobre el territorio, aún tratándose de un fenómeno de reciente activación, contamos con estudios sobre casos ya madurados, como son los efectos de saturación en el Valle del Silicio debido a la excesiva concentración de actividades económicas determinada por la accesibilidad a los sistemas de información, impidiendo el desarrollo paralelo de la calidad de vida MOLINI y CASTANYER (1987). O bien como el caso del Japón donde se produjo a finales de Ia década de los 80 un redireccionamiento en las líneas para la ubicación de parques tecnológicos en el Japón STÖHR (1987). Estos dos casos nos muestran el factor "acceso a servicios telemáticos" como un elemento ordenador del territorio.

Sin embargo la diferencia cualitativa existente entre el contexto de las telecomunicaciones en los años 80 y la situación actual ha incentivado las investigaciones con fines prospectivos por parte, no sólo de los profesionales de Ia cibemética e ingeniería de sistemas, sino también en el campo de la sociología, psicología DANET (I995) y otras ciencias que nunca habían sido identificadas con el desarrollo de la microelectrónica y la transmisión de datos ${ }^{1}$.

1. En el campo de las telecomunicaciones la palabra dato se entiende por información digitalizada y 
El cambio cualitativo experimentado reside en la inversión de la dinámica por la cual el desarrollo de los sistemas de información y herramientas para la gestión habían evolucionado de forma paralela al sector de la informática, de manera que las ofertas de los productos informáticos a los directivos de las empresas dependían del desarrollo del software y hardware en el momento (COOPER \& LYBRANDS 1993). Hoy día pocas empresas son capaces de aprovechar plenamente estas posibilidades, creando una situación de competencia en la que la estrategia de inversiones en infraestructura informática, telemática y la formación correspondiente para su aplicación, pueda determinar el balance final en la pugna por el mercado.

Esta revolución que estamos presenciando puede traer consigo impactos sobre el territorio a mayor escala incluso que la observada en el Valle del Silicio. Podemos referimos ya a la existencia de unos patrones diferentes que van a aportar un nuevo orden en la división internacional del trabajo y una nueva concepción del espacio en las actividades del hombre BAKIS (1995). Las NTI pueden traer nuevas soluciones para el desarrollo endógeno y el aislamiento crónico de determinadas regiones SEGUI PONS (1995) y se considera como pieza clave en algunos planes de desarrollo local POSADA y MORENO (1995). En el diseño de la red física y lógica española han intervenido de manera decisiva las administraciones públicas Central Autonómica y Municipal, incentivado en buena parte por la actuación de programas de la Unión Europea STAR, TELEMATICA, etc.

En Andalucía al igual que en el resto de España se han venido desarrollando diversos ambiciosos pIanes de infraestructuras animados por fondos nacionales y de la Unión Europea, que a su vez han ido generando o incentivando la creación de otros proyectos menores siguiendo siempre como criterio el ámbito de competencia de las instituciones implicadas. De este modo podemos ver por parte de los Ministerios de Educación y Ciencia, junto con el de Transportes y Comunicaciones como se implementa la red de Interconexión de Recursos Informáticos (IRIS) para el ámbito científico y académico a nivel nacional y, en la misma línea, el Plan de Informatización de la Administración de la Comunidad Andaluza (PIACA), que a su vez produjo el diseño de los Sistemas de Información Territorial, Medioambiental, Geológico Minero, etc., aparte de la red corporativa de la Junta de Andalucía.

Sin embargo la falta de precisión y planificación en la implementación de estos planes puede llevamos a situaciones negativas sobre el terreno y de naturaleza diferente a la que se diagnosticó en el Valle del Silicio y la política de parques tecnológicos en Japón durante la década de los 80. En el caso español, la inadecuación entre la infraestructura de los sistemas de información y el tejido empre-

viene expresada cuantitativamente en términos informáticos: Kbytes por segundo (KPS), megas por segundo (MPS). 
sarial por defecto del primero puede ahogar las actividades innovadoras del segundo Pero si es al contrario, esto es el caso de Andalucía, que las NTI pasan a la fase d $\epsilon$ implementación sobre unos tejidos productivos que aún no están en una fase Ic suficientemente madura como para asimilar los nuevos valores de la información o incluso la falta de receptividad por los agentes implicados, podemos crear une situación de desorden que prolongue la desintegración entre el tejido productivo y las NTI. GARCIA, MORENO y POSADA (1995).

\subsection{Aproximaciones al análisis topológico}

El desarrollo del componente físico de las telecomunicaciones, es decir, tantc el cableado como los equipos de transmisión ${ }^{2}$, ha recaído hasta el momento bajc la responsabilidad de la compañía TELEFONICA, cuya situación parece tan sólida como la que ostentaba antes del proceso de liberalización de las telecomunicaciones que se está produciendo en nuestro país. La aparición de nuevas empresas puede dinamizar el desarrollo del componente físico, pero la red de cableado actual, tendida y explotada por TELEFONICA casi en su totalidad, difícilmente tendrá una competencia sólida, en tanto en cuanto la liberalización llega cuando se ha alcanzado un nivel de desarrollo suficiente como para dar cobertura de redes de alta velocidad a todo el territorio nacional ${ }^{3}$.

Las diferentes administraciones han hecho circular sus sistemas de Información por esta red física partiendo de las redes troncales de IBERPAC e IBERCOM, que en el caso de Andalucía siguieron una distribución determinada por el sistema de ciudades de la Región como resultado de intervenciones tendentes a primar una forma de organización de las actividades económicas ESPAÑA RIOS (1994).

Este primer paso se basaba en el uso de las redes telefónicas conmutadas (RTC) ${ }^{4}$ con líneas punto a punto fundamentalmente. Posteriormente la aparición de la red

2. Actualmente se sigue una línea multimodal en la que participan tanto radioenlaces punto a punto como a través de líneas vía satélite, cañones láser, etc.

3. En palabras del Sr. León Martínez en el centro de demostraciones de TELEFÓNICA, la política actual de la compañía mantiene esta línea. Cualquier usuario, esté donde esté, podrá solicitar el acceso a las líneas de alta velocidad y los servicios integrados pagando la misma cuota independientemente de donde se encuentre. Con ello se evita la "penalización geográfica". En muchos casos las solicitudes no son rentables, pues así está contemplado en el contrato con el Estado, donde TELEFONICA se compromete a dar cobertura total al territorio nacional, pero con ello además se consigue una extensión territorial del mercado de TELEFONICA que ostentará una posición muy sólida frente a las nuevas compañías que intenten introducirse en el mercado concluida la situación de monopolio.

4. La primera modalidad en la transmisión de datos por cable fue a través de las redes telefónicas conmutadas, las cuales se basaban en un sistema por el cual la línea se mantenía activada por el usuario que estuviese transmitiendo. La conmutación digital de las líneas permitía el uso de diferentes estaciones dependiendo de las líneas disponibles más que de la trayectoria directa. De esta forma en una comunicación entre Sevilla y Alicante la conexión podía tener en servicio constante a estaciones tan distantes de Ia trayectoria 
IBERPAC facilitó la entrada en servicio de las Redes de Transmisión de Paquetes (RTP) y finalmente la Red Digital de Servicios Integrados (RDSI). Los inicios de las redes de transmisión crearon una jerarquización de los accesos telemáticos de manera que sólo las grandes empresas (bancos fundamentalmente) e instituciones de peso podían costear el mantenimiento de estas líneas. La aparición de IBERPAC (hoy IBERNET) y posteriormente la red con el protocolo X25 se facilitó el paso hacia la alta velocidad a la comunidad académica e investigadora. Actualmente la RDSI ha descendido de escala llegando a estar al alcance de las PYMEs, GARCIA, MORENO y POSADA (1995) e incluso de usuarios individuales. En lo que aquí nos concieme el aumento de cobertura potencial por su accesibilidad significa la completa distribución de las NTI pudiendo implicar a las administraciones locales. Es en este momento cuando las diputaciones y gobiernos regionales fundamentalmente deberían haber llegado a un consenso que repercutiese favorablemente en eI contribuyente, aspecto que se tratará más adelante, siendo la clave de este trabajo.

\subsection{Las NTI como herramienta de trabajo}

La informática y acceso a las autopistas de la información significa para eI geógrafo, como para cualquier otro profesional que tenga entre sus tareas la recogida y elaboración de datos, la facilidad de acceso a sistemas de información de contenido variado, estando la máxima expresión en eI desarrollo de SIGs acceso a bancos de datos remotos con contenido cartográfico, estadístico o incluso sensores, estaciones meteorológicas, etc. De hecho los planes más ambiciosos por el coste económico que supone el levantamiento y digitalización de la información que contienen los SIGs, son en Andalucía los Sistemas de Información Territorial, Medioambiental y Geológico-minero. Con ellos las NTI constituyen una vía de

como puede ser en teoría Sevilla-Córdoba-Badajoz-Toledo-Albacente-Alicante, o bien Sevilla-Córdoba-JaénMurcia-Alicante. Se trataba de una transmisión completamente analógica. La conmutación elige las rutas disponibles pero mantiene una línea en uso constante. En los casos de las grandes empresas podía mantener una comunicación digital a través de los servicios "punto a punto" IBERCOM, los cuales mantenían una línea al servicio de un usuario concreto. La revolución comenzó con la transmisión de datos por paquetes digitales (RTP) de manera que podían mandarse a través de una red en secuencias (paquetes) codificadas que reconstituían el mensaje completo al llegar a su destino. Esta conmutación sigue la misma lógica que la RTC en su trayectoria, la diferencia reside en que varios usuarios pueden estar utilizando Ia misma línea al mismo tiempo. La limitación de este tipo de transmisión es lo costoso de su instalación, por lo que su acceso estaba restringido a lugares e instituciones con gran peso específico. La RTC puede conectarse también a la RTP pero con las limitaciones ya explicadas para el último tramo. La aparición de la Red Digital de Servicios Integrados (RDSI) significó la posibilidad de transmitir información digital por frecuencias. Podría interpretarse como un resultado "híbrido" entre la RTP y la RTC con la facilidad de la transmisión digital que tiene la primera, con su misma capacidad de redireccionamiento, pero además con la capacidad de cobertura que corresponde al tendido de la RTC. Así los sistemas de información pueden ser accesibles a través de INFOVIA a la cual se accedería mediante conexión por RDSI. 
información ágil y actualizada para el análisis y gestión del territorio MORENC NAVARRO (1995). El estudio de su propia topología es posible dominando las técnicas de navegación, pudiendo incluso hacer de este modo un estudio del desarrollo de las autopistas de la información en zonas remotas. Incluso existe la posibilidad de facilitar la prospección de actuaciones ligadas a las telecomunicacio. nes, por lo que determinados trabajos de evaluación pueden realizarse desde unć estación de trabajo, mediante encuestas o entrevistas que pueden realizarse en vivc (on-line).

\section{LA GLOBALIZACIÓN DE LA INFORMACIÓN}

La revolución que estamos presenciando está siguiendo su trayectoria según e modelo que se está desarrollando en los EE.UU. La infraestructura nacional de información de los EE.UU. constará de cientos de redes separadas, manejadas po] compañías diferentes con tecnologías diferentes pero compatibles, conectadas er conjunto con una enorme red de redes que proporcionará video teléfonico y digita interactivo a casi todos los norteamericanos, es decir, la disponibilidad de estas infraestructuras estará al alcance de las escalas mínimas en los sectores de producción. Este plan se basará en cinco principios:

1․ Alentar a la empresa privada.

$2^{\mathrm{o}}$ Promover la competencia.

$3^{\text {o }}$ Crear un marco regulador flexible que pueda mantenerse a la par de lo: rápidos cambios de tecnología y el mercado.

4ํㅡㄹ Darles a todos los proveedores de información acceso abierto a la red

$5^{\text {o }}$ Asegurar la universalidad del servicio.

(AL GORE, 1994).

La cobertura de estos servicios puede ciertamente llegar a ser global a cortc plazo, por lo que los planes de desarrollo regional se encontrarían con un nuevc elemento de articulación territorial susceptible de producir, como se apuntó ante riormente, un cambio en las relaciones centro-periferia por la trascendencia que lt corresponde a una nueva revolución de las comunicaciones. Estas circunstancia: pueden provocar la generalización de un contexto ideal para la PYME, debido a abaratamiento de los costes en infraestructuras y aumento de la oferta del suelo Pero también puede crearse un nuevo desequilibrio por las ventajas mercantiles qut presentan las regiones más avanzadas en el sector de las telecomunicaciones. Ant estas evidencias es especialmente importante prestar atención al punto tres de lo: principios expuestos por AL GORE: la regulación del sector de las telecomunica ciones resulta fundamental para conseguir un desarrollo equilibrado. La existencia 
de una legislación avanzada al respecto puede significar el adelanto de esta en la lucha por el dominio del mercado de la información. El sector de las telecomunicaciones en España está constituido por una institución reguladora ubicada en el Ministerio de Obras Públicas, Transportes y Medio Ambiente, un grupo de operadores de red representados por Telefónica, Retevisión, Correos e Hispasat, un número de empresas suministradoras de equipos, instalaciones y un número creciente de pequeñas empresas proveedoras de servicios, la mayoría de las cuales acaba de entrar. Mientras los proveedores más recientes, de capital extranjero fundamentalmente se aprovecharon de la falta de regulación de estas explotaciones para establecerse.

En cuanto la provisión de servicios de telecomunicaciones comenzó a estar al alcance de compañías privadas como GOYA, COMPUSERVE y SERVICOM principalmente de capital extranjero, nos encontramos conque estaban cobrando al ciudadano por el acceso a unos sistemas de información que habían sido producido por las administraciones Central y Autonómicas, produciéndose un contrasentido cercano al fraude que resultaba casi inevitable. Actualmente los controles de seguridad puede mantener privilegios de acceso a los sistemas de información que no se desean poner a disposición del público, pero aquellos usuarios que estén dispuestos pagar el valor añadido que contiene la información que necesitan la pueden disponer solicitando el acceso. Este aspecto nos remite a la situación final del desarrollo de la topología de acceso expuesta en el punto 2.2.

En esta situación tiene necesariamente para participar las administraciones de manera que no existan agravios comparativos (penalizaciones geográficas) incluso fomentar el uso de estas tecnologías donde no han tenido la receptibilidad adecuada.

\section{LA TRAYECTORIA DE LAS ADMINISTRACIONES PÚBLICAS EN EL DESARROLLO DE LAS AUTOPISTAS DE LA INFORMACIÓN}

En este punto no se mantendrá un orden en el descenso de la escala, ya que las administraciones Central y regionales, en este caso Andalucía, comenzaron a trabajar sus respectivas redes antes de que entraran en vigor los planes de la Comunidad Europea STAR y TELEMATICA. Se atenderá preferentemente a un avance en el tiempo hasta llegar a las últimas ramificaciones que pertenecen a la escala local, pero que sin embargo han tenido un tratamiento especial desde la Comunidad Europea. En esta escala la que presenta mayor conflictividad, ya sea por lo escasamente significativas que están resultando ser las Diputaciones Provinciales, frente a las políticas regionales o bien por ser el ámbito local eI más cercano al ciudadano y por lo tanto la más proclive a manifestar sus perjuicios. 
También tendrán en este punto un especial tratamiento las redes académicas pues la implementación de las NTI fue planeada de manera que ésta madurase cor los recursos intelectuales en el ámbito académico para luego pasar al tejido pro. ductivo, el cual se muestra todavía reciente a adoptar las NTI (RODRÍGUEZ. ROSELLO 1994), aspecto que debe ser muy tenido en cuenta a la hora de empren. der planes con el fin de introducir estas tecnologías entre los agentes vinculados con dicho tejido.

\subsection{La transmisión de datos en España. Desde la RETD a la INTERNET}

El Ministerio de Transportes y Comunicaciones comenzó muy tempranament la configuración de una red de transmisión de datos por RTP. Inaugurada por le CTNE en 1971 fue la primera red de conmutación de paquetes en el mundc (CARBALLAR FALCON 1993).

Posteriormente, y con miras a la conexión internacional, se fueron adoptandc una serie de protocolos que fuesen compatibles con otras redes que se estabar desarrollando en el extranjero. Este período de desarrollo de protocolos lo podemos cerrar en torno a 1982 con la creación de la red IBERPAC y la adopción del protocolo más universal X25. Este protocolo se mantuvo vigente varios años posibilitando Ia transmisión de paquetes con casi todos los países del mundo desaпollado. La red estuvo disponible para todos los usuarios que la solicitasen, siempre que pudieran costear las tarifas de acceso y uso. NaturaImente la diferencia entre aquella y la situación actual estriba en que la X25 requería una gran inversión para el "enganche" que normalmente se circunscribía a áreas próximas a un anillo determinado. Esto estaba al alcance de grandes empresas y algunas instituciones del ámbito científico 5 .

A nivel académico bajo la dirección del Ministerio de Educación y Ciencia creć la red IRIS (Interconexión de Recursos Informáticos), la cual publicó su primer boletín en 1989. Este programa sufraga todos los gastos de uso de la red, por el momento, siendo una institución con labores más organizativas (logística) que de ingeniería. Este programa puso en funcionamiento la red ART1X que soportó y gestionó las redes académicas a nivel nacional hasta octubre de 1995 corriendo por la red física IBERPAC. En esta fecha se considera concluida una etapa llamada "de producción" (MORENO, J.C. y RINCON, M. 1996) por lo cual se decide cambiar la ubicación del nodo central de ARTIX, desde un centro docente, la Escuela Superior de Ingenieros de Telecomunicaciones en Madrid, para colocarlo en un centro propio de la organización, pasando a ser el nodo central SIDERAL.

5. La otra alternativa era el servicio IBERCOM ya expuesto con anterioridad, circulaba por RTC "punto a punto", o en términos más asequibles, se trataba de una línea directa alquilada por determinadas empresas. 
En la trayectoria de la red IRIS en la INTERNET merece destacar los siguientes hitos.

\section{CUADRO I}

Julio de 1990

Conexión de España a la INTERNET.

Diciembre de 1990

Conexión de los primeros cuatro centros (CICA, DIT, FUNDESCO y CIEMAT).

Marzo de 1991

Se inicia la primera fase operativa de SIDERAL.

Octubre de 1991

Primeras 1.000 máquinas conectadas.

Enero de 1992

Se crea el primer servidor comercial ${ }^{6}$.

Agosto de 1993

Se llega a 10.000 máquinas conectadas.

Mayo de 1994

Se llega a 20.000 máquinas conectadas y 100 organizaciones.

Octubre de 1995 Migración de ARTIX a SIDERAL.

Fuente: Grupo de Comunicaciones del CICA.

\subsection{El PIan Informático de la Administración de la Comunidad Andaluza (PIACA)}

Desde que en 1984 comenzara la implementación de este Plan de Informatización, se han hecho grandes esfuerzos para dotar a la región de una infraestructura moderna e integrada capaz de gestionar las labores que eran competencia de la Comunidad. En 1985 comenzaron a crearse los grandes sistemas horizontales y las planificaciones sectoriales. Los primeros se dedicaron a la gestión de personal y contabilidad y los segundos han dado como fruto los sistemas y redes de transmisión de datos, objeto principal del presente trabajo.

El PIACA se compartimentó en cinco etapas temporales ya concluidas que son:

6. Se trata del primer proveedor privado. La irrupción de estos proveedores comienza a atraer la atención de determinados empresarios y profesionales, posiblemente estimulados por la desmedida campaña propagandística sobre las excelencias de la INTERNET. Por otra parte podemos considerarlo como un buen tratamiento de choque contra esa falta de receptividad advertida por RODRIGUEZ ROSELLO. 
$1^{\circ}$ 1984-1985. La incorporación de la informática.

$2^{\text {o }}$ 1985-1987. El desarrollo de los grandes sistemas horizontales y las planifi. caciones sectoriales.

3 1987-1988. La extensión de la microinformática, la ofimática y la homolo. gación de productos.

$4^{0}$ 1989-1991. Las planificaciones anuales y el impulso al desarrollo del software de aplicación, los sistemas de información al ciudadano.

50 1991-1992. Los compromisos con los estándares y la convergencia con las otras administraciones?

Fuente: Junta de Andalucía. Consejería de Gobernación (1993).

Dentro de este cronograma se pusieron en funcionamiento los siguientes planes informáticos.

- Plan Informático de la Agencia del Medio Ambiente.

- Plan Informático de la Consejería de Economía y Hacienda.

- Plan Informático del Instituto Andaluz de Reforma Agraria (IARA).

- Plan Informático de los Centros de Seguridad e Higiene en el Trabajo de la Consejería de Trabajo.

- Plan Informático de la Consejería de Educación y Ciencia.

De estos planes merecen especial atención las siguientes actuaciones:

a) El sistema de Información Hospitalaria de Andalucía cuyo fin es el de establecer en todos los centros de la Comunidad Autónoma un sistema de información homogéneo a través de la informatización de las áreas Administrativo-clínica, Personal, Contabilidad, Almacenes, Farmacia y Suministro y Mantenimiento.

b) Creación de los Sistemas de Información Medioambiental (SINAMBA) dependiente de la Agencia del Medio Ambiente (hoy Consejería de Medio Ambiente), e Información Geológico-Minero (SIGMA) dependiente de la Consejería de Economía y Hacienda.

c) La creación de la Red Informática Científica de Andalucía (RICA) en 1985 y el Centro Informático Científico de Andalucía (CICA) en 1987.

Esta actuación es Ia más relevante a la hora de explicar el desarrollo de Ias autopistas de la Información en Andalucía. Su objetivo fue el de dotar a la comunidad científica y académica de Andalucía de una infraestructura de red informática y optimizar de este modo la compartición de recursos. En un principio esta

7. Sobre los puntos 4 y 5 se tratará más adelante por estar comprometidos con los programas STAR, TELEMATICA y por la referencia a las "otras administraciones". 
red soportaba los protocolos de comunicación DECNET y XODIAC dividiendo Andalucía occidental de la oriental respectivamente. El enganche de RICA a la red ARTIX como red de alta velocidad, adopta el protocolo X25 con el que consigue el acceso a las redes internacionales. En 1987 la creación del CICA se convierte en el primer nodo externo de la red ARTIX, la cual en 1990 sólo conecta al mencionado Centro Andaluz y a FUNDESCO, DIT y CIEMAT. Desde este momento las redes académicas a nivel nacional comienzan a proliferar dando fuerza a la filosofía de INTERNET provocando la irrupción de nodos proveedores de información privados con las consecuencias señaladas en el Cuadro 1.

\section{LA PARTICIPACIÓN DE LA COMUNIDAD EUROPEA: DE LOS PROGRAMAS STAR Y TELEMÁTICA HASTA EL GRUPO TEN-34 ${ }^{8}$}

El programa STAR estaba planteado desde el Consejo de la Comunidad Europea, aunque eran los estados beneficiados los que tenían que elaborar los programas de intervención específicos. Los programas españoles se aprobó en octubre de 1987 con una inversión que superaba los 60.000 millones de pts. de los cuales 15.787 (casi un $28 \%$ ) fueron a parar a Andalucía por ser región de objetivo 1 (JORDA BORRELL 1992). Este programa se dividía en dos grandes apartados, uno dedicado a infraestructuras y otro destinado al fomento de los Servicios Avanzados de Telecomunicaciones. Con esta inversión se intentó aproximar estos servicios a las PYMEs con la creación de Oficinas de Servicios de Telecomunicaciones (OSITs), cuestión que se tratará más adelante, ya que estos planes descienden su ámbito de implementación a la escala comarcal.

El programa TELEMATICA fue una continuidad del programa STAR con la diferencia de que sólo se dedicaría al desarrollo de los SATs, sin tratar el tema de infraestructuras. Varias instituciones de educación y ciencia, compañías y administraciones de los entonces doce estados miembros de la Comunidad Europea llevaron a cabo una serie de programas I+D con el objetivo de estimular la creatividad y sobre todo establecer una base para continuar prosperando en el siglo XXI. Se habrían de Jacques Delors en su investidura como Presidente del Parlamento Europeo (10-2-1993) anunció: "desarrollaremos programas que creen una gran red de infraestructura de transporte, telecomunicaciones, bases de datos y formación".

El programa de I+D creado por la Comisión Europea llamado "Sistemas Telemáticos" en áreas de interés general tiene los objetivos marcados a largo plazo. Contiene siete subprogramas en áreas que se han mostrado como potenciales demandantes de las NTI:

8. El grupo TEN-34 se constituyó después del cambio de nombre de Comunidad Europea a Unión Europea. 
1. Intercambio de Información entre las administraciones europeas.

2. Servicios de transporte.

3. Sanidad.

4. Educación flexible a distancia.

5. Bibliotecas.

6. Lenguaje de programación.

7. Sistemas telemáticos para áreas rurales.

Estas siete áreas atrayeron el interés de pequeñas y grandes proveedores de NTI operadores de redes, universidades, centros de investigación y numerosas compa. ñías, principalmente dentro del rango de las PYMES. Los programas tendrían que canalizarse por las Administraciones Centrales de cada país, redistribuyendo su pesc en instituciones públicas y privadas, cooperando éstas con un $50 \%$ del total de $1 \varepsilon$ financiación. Todo esto justo antes de que nos encontrásemos con la situación er la que ya hay varios operadores y servidores de información telemática en España

El proyecto TEN-34 se encuentra todavía en una fase primaria de desarrollo. por lo que no podemos tener una información muy elaborada al respecto. En enero de 1995 se formó el grupo de gestión Stering Group como órgano director para la organización del proyecto TEN-34, donde la anteriormente mencionada red IRIS y TELEFONICA estaban representados. Se encargó a DANTE la redacción de una propuesta a partir del estudio de viabilidad que redactaría EUROCAIRN para posteriormente presentarla a la Comisión. A mediados de 1995 se entregó el proyecto firmado inicialmente por los siguientes países: Alemania, Austria, Francia, Gran Bretaña, España, Luxemburgo, Portugal, Suecia, Dinamarca, Holanda, Suiza y Bélgica. Paralelamente comenzaron las sesiones técnicas para fijar los requisitos que tendría la futura "Red Paneuropea". Al principio se diseñó una red en forma de "nube" (muy distribuida por el territorio y sin organización troncal significativa) formada por líneas punto a punto en la que cada país tendría una velocidad de 34 Mbs $^{9}$. Posteriormente se pidieron propuestas siendo la más sólida la del grupo UNISOURCE, consorcio intencional en el que participa TELEFONICA. Pero no todos se pusieron de acuerdo, España no llegó a confirmar su participación hasta el 13 de marzo de 1996. El estado actual del proyecto es el siguiente:

- La línea de 34 Mbs se valorará entorno a tres millones de Ecus.

- La subvención contemplaba 15 meses de servicio como prueba previa.

- Se adjudicada el servicio a UNISOURCE y FUDI.

- La subvención de la Unión Europea será de un $40 \%$.

- Los países firmantes deberían realizar un acuerdo financiero.

- El proyecto se iniciaría durante el primer trimestre de 1996 y el primer servicio se daría en octubre de 1996.

9. Para hacernos una idea, actualmente la transmisión troncal en España se mueve en tomo a los 2 Mbs. 
Naturalmente podemos intuir que se trata de un proyecto de infraestructura $y$ gestión para aumentar las prestaciones de una tecnología que se considera lo suficientemente difundida.

\section{LAS REPERCUSIONES A ESCALA LOCAL EN ANDALUCÍA: LA TRAYECTORIA DE LAS DIPUTACIONES}

Los estudios que se han realizado sobre el sector de las telecomunicaciones en Andalucía han confirmado el bajo grado de penetración de los STAs, constituyendo un grave obstáculo para el acercamiento de nuestra comunidad al resto de Ias regiones europeas (MIRANDA BONILLA 1995). No se ha advertido la profundización a la que aspiraban los programas STAR y TELEMATICA estando este último especialmente interesado por las áreas rurales. De las ocho divisiones y empresas informáticas correspondientes a cada una de las diputaciones de la Comunidad Andaluza, tan sólo la de Sevilla se ha visto beneficiada directamente de estos programas europeos presentando sus proyectos en solitario.

\subsection{La Diputación de Sevilla: IMPRO, S.A.}

Además del apoyo de los programas europeos STAR y TELEMATICA se contó en un principio con una posible financiación con fondos FEDER para el desarrollo de un Sistema de Información Geográfico. Para invertir los fondos provenientes del programa STAR se creó el grupo ORTELIUS con la intención de definir la estrategia de la Diputación respecto a la distribución de la información por medios telemáticos. Este grupo resultó redundante cuando se puso en marcha el programa TELEMATICA, el cual transformó el Centro Servidor de Videotex existente, creado con anterioridad por el proyecto ARTIDE, en un centro de comunicaciones videotex para toda la provincia. A lo largo de 1993 se mantuvieron reuniones para configurar la Red Telemática Provincial, con la puesta en funcionamiento de las OSITs y el proyecto ALTEX, consistente en la distribución de 3.000 terminales videotex. Este programa sufrió un duro revés por reducciones presupuestarias en 1993, rompiendo la dinámica iniciada por el proyecto STAR e impidió la justificación de la inversión de unos fondos ya comprometidos. El trabajo de campo en diversos ayuntamientos de la Sierra Norte de Sevilla y en la Mancomunidad del Bajo Guadalquivir evidenciaron el hecho de que el proyecto de red telemática no había sido recibido con el entusiasmo que hoy día podemos ver por las NTI. Quizás se trató de un adentro que ha resultado contraproducente, requiriendo ahora una remodelación de las directrices y el diseño de una nueva infraestructura. Esto iría en la línea de otras diputaciones de la región. 


\subsection{La Diputación de Córdoba: EPRINSA}

Parece ser la más independiente de las diputaciones en lo que a organización : diseño de planes se refiere. Se partió de la idea de unificar todos los ayuntamien tos bajo una red con sistema operativo UNIX. Con este propósito se puso en mar cha el proyecto TELEMUN 1994, el cual tiene hasta el momento un fuerte nive de implantación, siempre según el responsable de funciones externas de la empre sa Sr. D. Manuel Roldán. Actualmente cuentan con una red corporativa para la: oficinas de la Diputación (algo común a todas las diputaciones de Andalucía)! han conseguido que algunos ayuntamientos se conecten vía modem con las ofici nas recaudatorias. Actualmente se están desarrollando nuevas aplicaciones para quধ los ayuntamientos conectados puedan acceder al Padrón, gestión Tributaria y Con tabilidad Fiscal.

Actualmente se encuentran evaluando los resultados y se ha advertido el mis mo caso de reticencia por parte de los ayuntamientos a la hora de introducir la: NTI en sus labores de gestión municipal.

\subsection{Servicio de Informática de la Diputación de Jaén}

Jaén es la más aislada de las provincias en lo que a desarrollo telemático sє refiere. Muy comprometida con los contratos a otras empresas, la Diputación pa. rece estar bastante alejada de desarrollar programas propios de desarrollo de NT. como los que hasta ahora se han expuesto. Se ha provisto a los ayuntamientos más pequeños de software y formación, como testimonio de la actuación de la admi. nistración provincial, pero siempre dejando este aspecto en manos de empresas privadas.

\subsection{El proyecto Indalo}

Las otras cinco provincias, Almería, Cádiz, Granada, Huelva y Málaga estár integradas dentro del grupo INDALO. Este grupo se inició advirtiendo la necesidad de homogeneizar los patrones que deben dirigir las redes de información, pou lo que decidieron reunirse, quizás un poco tardíamente, pero con grandes perspectivas y confianza en el proyecto conjunto. El germen de este trabajo surgió en unc reunión técnica celebrada en La Rábida, lugar que dio nombre al primer grupo frutc del compromiso que allí se gestó sobre dicha necesidad. Apoyándose en los programas ARCO y PASO que se habían elaborado y presentado por el gobierno cen. tral con el fin de obtener financiación de los proyectos europeos STAR TELEMATICA y de los fondos FEDER, para la elaboración de un sistema de in- 
formación para la administración local. En 1994 esa iniciativa, según el Director de los Sistemas de Información de la Diputación de Huelva Sr. Tomás Quintana, fue presentada a la Junta de Andalucía para que lo Iiderara incluyendo a las demás provincias sin haber tenido respuesta hasta el momento.

Finalmente el Ministerio para las Administraciones Públicas se encargó de ese liderazgo incluyendo también a la Asociación Navarra de Informática Municipal (ANIMSA). La Comisión Nacional para la Cooperación entre las Administraciones Públicas en el campo de los sistemas y tecnologías de la Información (COAXI) acordó en su reunión del 3 de febrero de 1994 constituir el grupo de trabajo para la elaboración del modelo de datos para el Intercambio de Información entre las Administraciones Locales (INDALO).

En este grupo se han integrado más de 250 profesionales de los distintos sectores de actividad representando a más de 100 entidades de las administraciones Central, Autonómicas y Locales (entre las que no se encuentra la Junta de Andalucía), que han trabajado en la elaboración del modelo de las áreas de Régimen Interno, Población, Gestión Económica, Contratación, Gestión Fiscal, Territorio, Protección Ciudadana, Servicios Sociales y Gestión Documental. En esta división del trabajo han tenido un papel predominante las Diputaciones de Cádiz y Huelva como emprendedoras del proyecto.

Hasta el momento se ha conseguido la comunicación por modem con todos los ayuntamientos de cada una de las cinco provincias mencionadas, excepto Granada que tiene un total de 150 en red de los 187 y Málaga que se encuentra algo rezagada en la definición de la red. En Huelva los municipios que lo solicitan se les instala sistemas muItiusuarios UNIX. Lo más importante es que la receptividad e incluso demanda por parte de los ayuntamientos está alcanzando un nivel prometedor, рог lo que podríamos encontramos con una auténtica red telemática que llegase a cubrir el territorio hasta la escala local.

\section{BIBLIOGRAFÍA}

BAKIS, E. (1995). "Telecommunications et territories: evolution de la problematique et perspectives". NETCOM. vol. 8, $\mathrm{n}^{\mathrm{9}} 2$. Octubre 1994.

BELLOSO, E. y ESPAÑA ROSI, I. (1995). "El prograsivo avance hacia la liberalización de las telecomunicaciones en España". Actas II Encuentro de Geografía Regional. La Rábida 1995, pp. 237-246.

CONSEJERÍA DE GOBERNACIÓN (1993). "La informática en la Junta de Andalucía”. Ed. Junta de Andalucía, Sevilla 1993, 160 pp.

COOPER \& LYBRANDS (1993). "Sistemas de información a la dirección". Como mejorar la gestión económico-financiera de la empresa. Madrid, vol. $\mathrm{n}^{\circ} 4$.

DANET, B. (1995). "Social Networking through Internet". Commision on communication networks and telecommunications. Annual meeting, Jerusalem 1995. 
ESPAÑA RIOS (1994). "La articulación de un nuevo orden territorial en la C.A. de Andalucía a partir del actual sistema de telecomunicaciones". Boletin de la AGE. $2^{\circ}$ semestre 1994, pF 27-44.

GARCIA, A.; MORENO, J. y POSADA, J. (1995). "Impacto de las NTI en el Desarrollo Regio nal”. Actas II Encuentro de Geografia Regional. La Rábida, 1995, pp. 219-228.

JORDA BORRELL, R. (1992). Las relaciones ciencia-tecnología-industria y el papel de l. administración. Instituto de Desarrollo Regional. Sevilla, 1992. 263 pp.

MOLINI, F. y CASTANYER. "Planeamiento territorial, administración Pública y Nuevas Tec nologías". Estudios territoriales. № 23, pp. 29-43.

MORENO, J. y RINCON, M. (1996). "El proyecto TEN-34". Boletín de la red IRIS. Abril d 1996, pp. 5-10.

MORENO NAVARRO, J. (1995) "The integration of a Regional Information System in telemati network of Andalusia". NETCOM. vol. 10 Janvier 1996, pp. 152-161.

POSADA, J. y MORENO, J. Nuevas tecnologías de la Información y desarrollo local. Evalua ción de impactos en espacios rurales sevillanos. Actas del XIV Congreso Nacional de Geo grafia. Salamanca, 1995, pp. 220-224.

RODIGUEZ ROSELLO (1994). Boletín de la red IRIS, $\mathrm{n}^{2} 29-30$, Diciembre 1994, pp. 58-72 SEGUI PONS (1995). "Information Networks in Mediterranean Countries: an alternative to re gional isolation. The Balearic Islands". NETCOM. vol. nº 10 Janvier 1996, pp. 231-298 